\title{
A Rolling Simulation System for Reversing Rolling Mills
}

\author{
Umut Hanoglu ${ }^{1,2, a}$ and Božidar Šarler ${ }^{1,2}$ \\ ${ }^{1}$ Laboratory for Simulation of Materials and Processes, Institute of Metals and Technology, Lepi pot 11, 1000 Ljubljana, Slovenia \\ ${ }^{2}$ Laboratory for Fluid Dynamics and Thermodynamics, Faculty of Mechanical Engineering, University of Ljubljana, Aškerčeva 6, 1000 \\ Ljubljana, Slovenia
}

\begin{abstract}
In this work a rolling simulation system has been developed for rolling schedules which consists of multiple reversing rolling mills. A slice model approach is applied where the position of a slice can only be determined by considering total deformation. Each slice is parallel to each other and perpendicular to the rolling direction. The solution of coupled thermal and mechanical models over each slice, at a given time and position, are achieved by a novel meshless Local Radial Basis Function Collocation Method (LRBFCM). Mechanical material model obeys ideal plastic flow rule defined by Von Mises. Unknown fields over the slices are interpolated by a certain number of collocation points distributed over the physical domain and its boundary. A system of equations is solved for each collocation point considering its local neighbouring points in the range between 5 and 7. A non-linear system of equations is solved by direct iteration. Groove geometries of each roll are implemented in a compatible way with the slice model and every roll has a horizontal orientation. In between each rolling pass the billet is rotated either 90 or 45 degrees clockwise or counter clockwise. Reduction at each of the passes can be very high, and in such cases, the material completely fills up the groove. This requires a special attention regarding the contact boundary conditions and the collocation node distribution due to numerical instability issues. Coulomb model of friction or sticking boundary conditions are used at the contact boundaries and Gauss-Seidel iterative elliptic node generation algorithm is used for redistributing collocation nodes over the physical domain, when necessary. The simulation results for arbitrary initial position of the slice in the billet are shown in terms of temperature, displacement, strain and stress fields as well as roll forces and torques. A user friendly computer application is created for industrial use based on C\# and .NET.
\end{abstract}

\section{Introduction}

In this paper a rolling simulation model is explained for reversing rolling mills. A steel billet is passed through a rolling stand multiple times and at each time the direction is reversed. At each pass, a different groove is used for shaping the material. In between the passes the billet is rotated either 90 or 45 degrees. The rotation is necessary since the mill has exclusively horizontal orientation. At each pass the billet becomes longer and takes more time to go through next rolling. The model expressed here is capable of simulating user defined reversing rolling mill uninterruptedly from the beginning until the end.

Simulation of rolling is in general very hard task of computational solid mechanics [1,2]. The complex groove geometries together with the deformed billet are reduced to $2 \mathrm{D}$ computational domains during present simulations. A meshless solution procedure is used together with the slice model assumption explained below. The Local Radial Basis function Collocation Method (LRBFCM) is used for interpolation of all the unknown fields over each slice at given time and position. LRBFCM has been previously described [3], applied to simulation of fluid flow problems [4], thermo- mechanics [5], dendritic growth [6], finishing rolling mills [7]. The rest of the paper is organized as follows. The physical model and the meshless solution procedure of the rolling simulation system based on both thermal and mechanical models are explained. Later the preferred node generation algorithm is elaborated. At the end, numerical simulation results of a reversing rolling mill with ten passes are shown and concluding remarks are given.

\section{Physical model}

In this research two major models are solved in a coupled way. Both thermal and mechanical models are defined in their strong form. Temperature field is obtained from the thermal model by solving it by explicit time stepping. The mechanical model is solved to get displacement, stress and strain fields.

\subsection{Slice model assumption}

The thermo-mechanical simulation of reversing rolling mill is a complex $3 \mathrm{D}$ process however, a simplified $2 \mathrm{D}$

\footnotetext{
${ }^{\mathrm{a}}$ Corresponding author: umut.hanoglu@imt.si
} 
modelling assumption may be introduced here based on slices aligned in parallel and towards the rolling direction as shown in Fig. 1. Each slice becomes a computational domain at given position and time. Slice is rolled multiple times until the end of the simulation and rotates in between rolls.

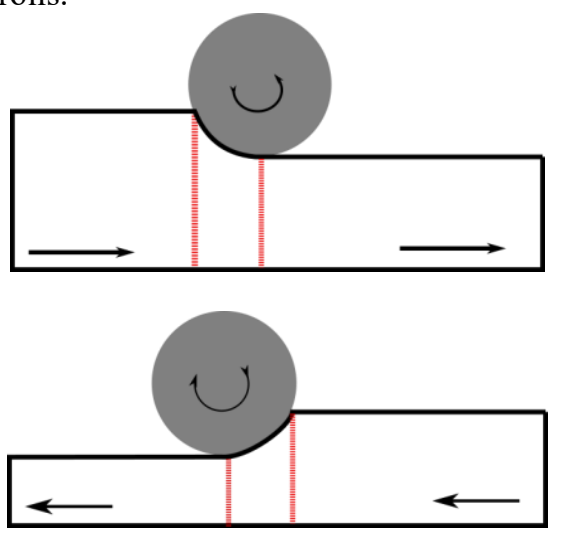

Figure 1. Computational slice positions and traveling of a slice through reversing rolling mill.

\subsection{Thermal model}

The temperature field is obtained from the following heat transfer equation applicable for slice model assumption

$\rho c_{p} \frac{\partial T}{\partial t}=\nabla \cdot(k \nabla T)+\dot{Q}$,

where $\rho, c_{p}, T, t, k, \dot{Q}$ stand for density, specific heat, temperature, time, thermal conductivity and internal heat generation rate due to deformation which is defined as

$\dot{Q}=\eta \frac{\partial}{\partial t} \int_{\bar{\varepsilon}} \bar{\sigma} d \bar{\varepsilon}$

where $\eta$ is Taylor-Quinney parameter, $\bar{\sigma}$ is effective stress and is $\bar{\varepsilon}$ effective strain. Robin type of boundary condition is applied over all of the boundaries.

$-k \frac{\partial T}{\partial \mathbf{n}_{\Gamma}}=h\left(T-T^{r e f}\right)$.

When there is a contact with the roll, the heat transfer coefficient $h$ is chosen much larger than to the air.

\subsection{Mechanical model}

For each slice in contact with the roll, the displacement field is solved directly from the governing equation used for the mechanical model.

$\mathbf{L}^{\mathrm{T}} \boldsymbol{\sigma}+\mathbf{b}=\mathbf{0}$

where $\mathbf{L}$ is the derivative operator matrix, $\boldsymbol{\sigma}=\left[\sigma_{x x}, \sigma_{y y}, \sigma_{x y}\right]^{\mathrm{T}}$ is the stress vector, and $\mathbf{b}=\left[b_{x}, b_{y}\right]^{\mathrm{T}}$ is the body force vector, however it is neglected in the current model. Essential and natural boundary conditions, used over the boundaries depending on whether the prescribed displacement $\overline{\mathbf{u}}$ or traction $\overline{\boldsymbol{\tau}}$ is known

$\overline{\mathbf{u}}=\mathbf{u}, \overline{\boldsymbol{\tau}}=\mathbf{n} \boldsymbol{\sigma}$

\section{Meshless solution procedure}

Instead of grid generation over the physical domain such as in FEM, collocations nodes without any geometrical shapes between them are used over the domain and boundaries. An example of an unknown field $\theta(\mathbf{p})$ at position $\mathbf{p}$ is interpolated with multi-quadric $\operatorname{RBF} \psi(\mathbf{p})$ over neighbouring nodes $(N=5,7)$ together with the augmented first order $\left(N_{p}=3\right)$ polynomial functions $\vartheta(\mathbf{p})$,

$$
\theta(\mathbf{p})=\sum_{i=1}^{N} \psi_{i}(\mathbf{p}) \alpha_{i}+\sum_{i=1}^{N_{p}} \vartheta_{i}(\mathbf{p}) \alpha_{N+i} .
$$

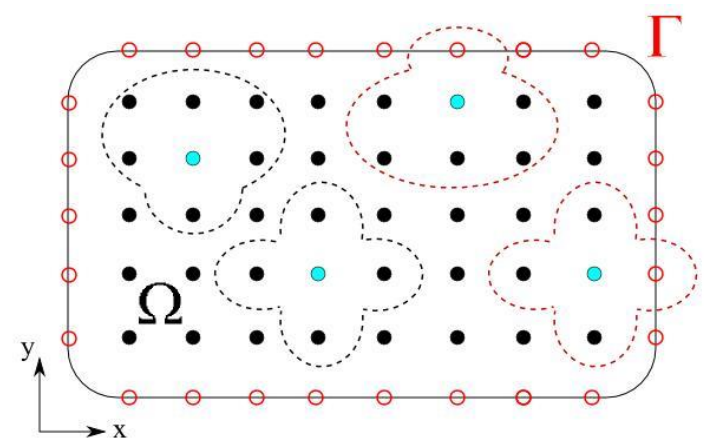

Figure 2. Examples of 5 or 7 noded local influence domians considered in the meshless solution.

\subsection{Solution of the thermal and mechanical models}

For the solution of the temperature field, the heat transfer equation is considered in the following discrete form

$\rho c_{p} \frac{T_{i}-T_{i 0}}{\Delta t}=\nabla \cdot(k \nabla T)+\frac{\Delta Q}{\Delta t}$.

Then the temperature field is interpolated locally for each collocation node considering only its neighbours.

$T(\mathbf{p})=\sum_{i=1}^{N} \psi_{i}(\mathbf{p})_{T} \alpha_{i}+\sum_{i=1}^{N_{p}} \vartheta_{i}(\mathbf{p})_{T} \alpha_{N+i}$

Temperature field in the governing equation and boundary conditions is discretized in this way and same collocation constants are used to obtain the temperature field. 
The governing equation used in the mechanical model may be written in terms of displacement vector $\mathbf{u}$ with using stiffness matrix $\mathbf{C}$, written in terms of effective stress-effective strain rate $(\bar{\sigma} / \bar{\varepsilon})$, explained in [8],

\section{$\mathbf{L}^{\mathrm{T}} \mathbf{C L u}+\mathbf{b}=\mathbf{0}$.}

The displacement vector $\mathbf{u}=\left[u_{x}, u_{y}\right]^{\mathrm{T}}$ components are interpolated in a similar way as the temperature field

$$
\begin{aligned}
& u_{x}(\mathbf{p})=\sum_{i=1}^{N} \psi_{i}(\mathbf{p})_{u_{x}} \alpha_{i}+\sum_{i=1}^{N_{p}} \vartheta_{i}(\mathbf{p})_{u_{x}} \alpha_{N+i}, \\
& u_{y}(\mathbf{p})=\sum_{i=1}^{N} \psi_{i}(\mathbf{p})_{u_{y}} \alpha_{i}+\sum_{i=1}^{N_{p}} \vartheta_{i}(\mathbf{p})_{u_{y}} \alpha_{N+i} .
\end{aligned}
$$

The system of equations are written in global form for each node to obtain the solution of displacements $(\mathbf{U})$ by direct iteration,

$$
\mathbf{K}\left(\mathbf{U}^{j-1}\right) \mathbf{U}^{j}=\mathbf{f},
$$

where $\mathbf{K}(\mathbf{U})$ is the global sparse matrix and $\mathbf{f}$ is the adjacent vector.

\subsection{Node generation algorithm}

The initial node configuration has an important role during the rolling simulation. If the initial size is a rectangle then a uniform node arrangement is applied at the beginning. If the initial slice has a unique nonrectangular shape then the nodes are distributed depending on iterative Gauss-Seidel elliptic node generation algorithm [9] as shown in Fig. 3.b.

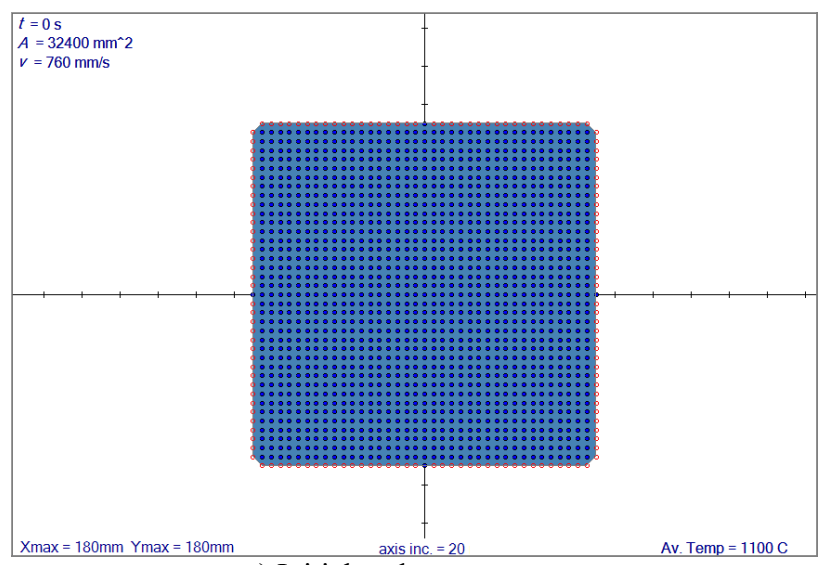

a) Initial node arrangement

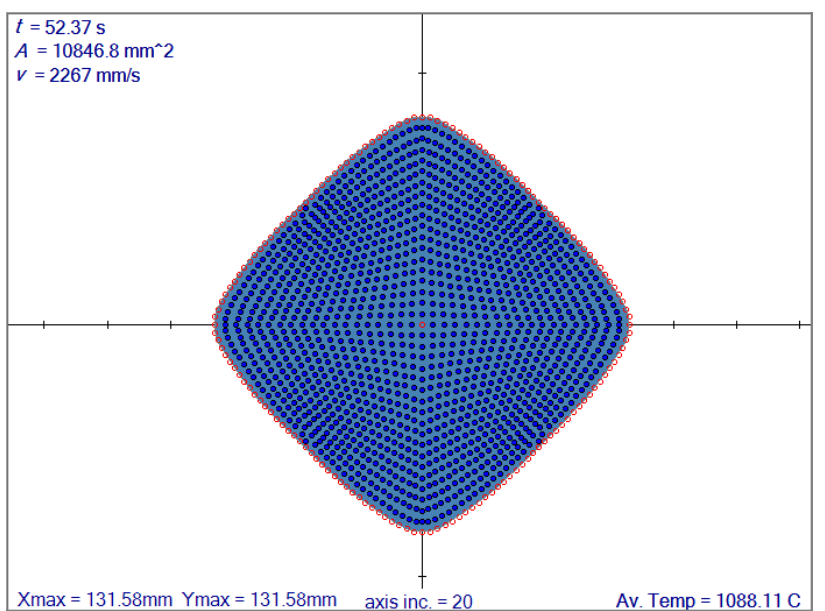

b) node arrangement after 45 degrees rotation

Figure 3. 1517 collocation nodes are shown in each figure. First figure shows the initial configuration, second figure shows the configuration after 45 degrees rotation before the second pass.

\subsection{Development of the simulation code}

A simulation code has been written from scratch using Microsoft Visual Studio C\# 2015 Express Edition [10]. A Windows desktop application is created with user friendly interface by using features of the Microsoft .NET framework. The only additional library used in this simulation code is Math.Net for solving the system of equations. The code is able to read multiple rolling schedules written in an excel file with all the involved groove types. Each groove is described based on 2D coordinates of multiple groove surface points written in a txt file. The simulation then calculates and redraws the corresponding groove line at each slice position and updates the boundary conditions accordingly. The user is able to change the slice position to any desired value and view all the results belonging to that slice.

\section{Simulation results}

Two different reversing rolling mills are used in the numerical simulations. During the first 5 passes the roll diameter is $800 \mathrm{~mm}$ and later for the subsequent 5 passes it is used as $650 \mathrm{~mm}$. The material model used is $\bar{\sigma}=589 \bar{\varepsilon}^{0.214} \exp (R(1200-T)) \mathrm{MPa}$ and the coefficient of friction is taken as 0.07 .

\subsection{Reversing rolling mill simulation with 800 $\mathrm{mm}$ roll diameter}

The initial shape and the node arrangement is used as shown in Fig. 3.a results of the first 5 passes are shown with groove slice lines drawn from the first contact until the last in Fig. 4. 


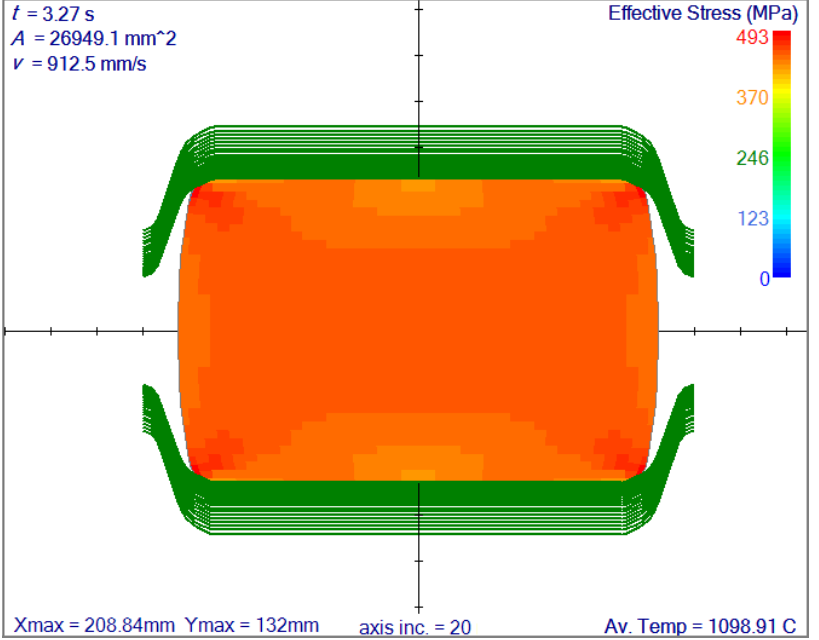

a) $1^{\text {st }}$ pass

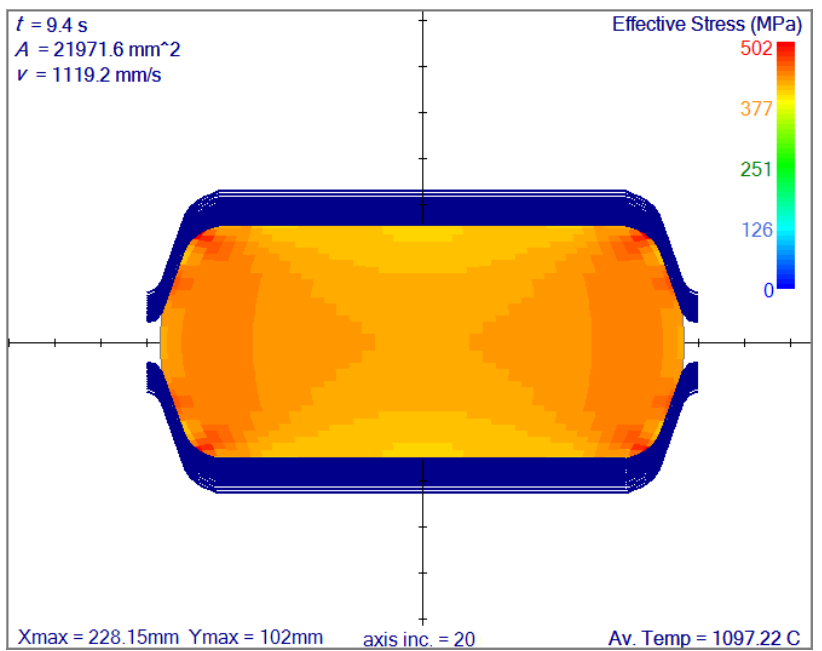

b) $2^{\text {nd }}$ pass

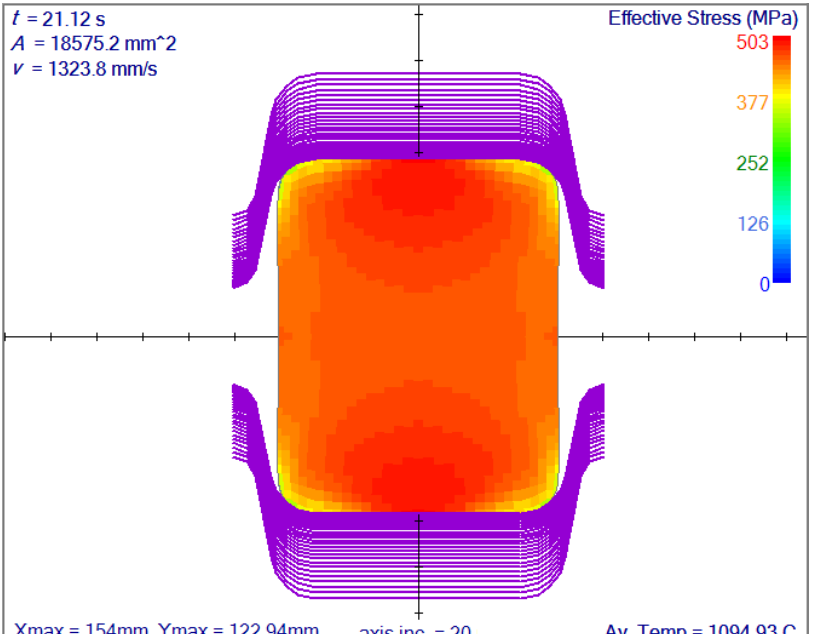

c) $3^{\text {rd }}$ pass

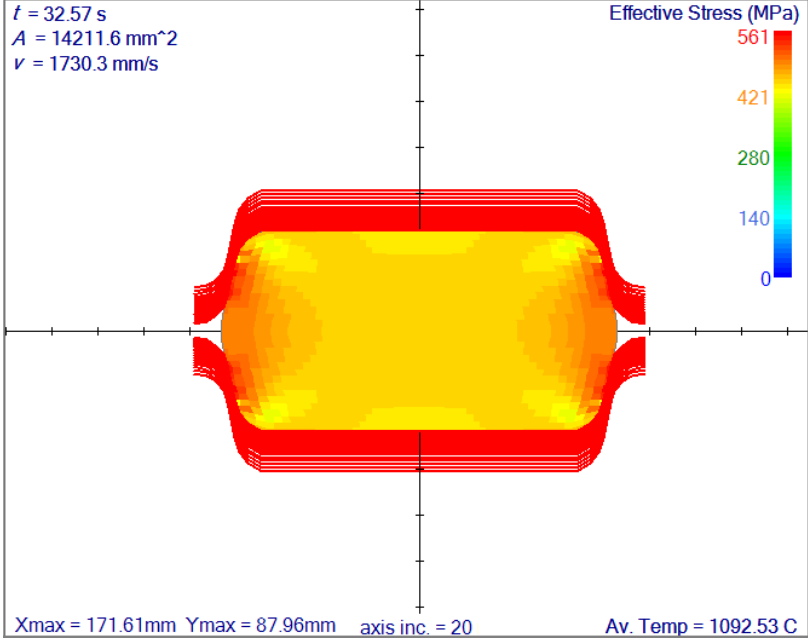

d) $4^{\text {th }}$ pass

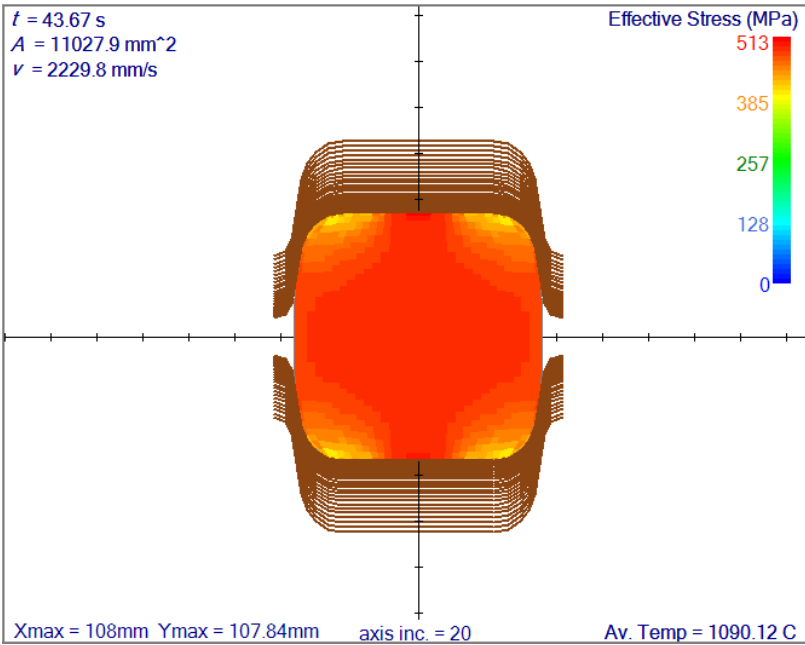

e) $5^{\text {th }}$ pass

Figure 4. Effective stress filed resulting after the first 5 passes when the billet is rotated 90 degrees after each pass starting from the second pass.

\subsection{Reversing rolling mill simulation with 650} $\mathrm{mm}$ roll diameter

This part of the simulation is a continuation of the previous simulation shown in Fig. 4. The slice in Fig 3.b is used for the initial slice shape and node arrangement. It has the same size as the slice shown in Fig. 4.e, but it is rotated 45 degrees. 


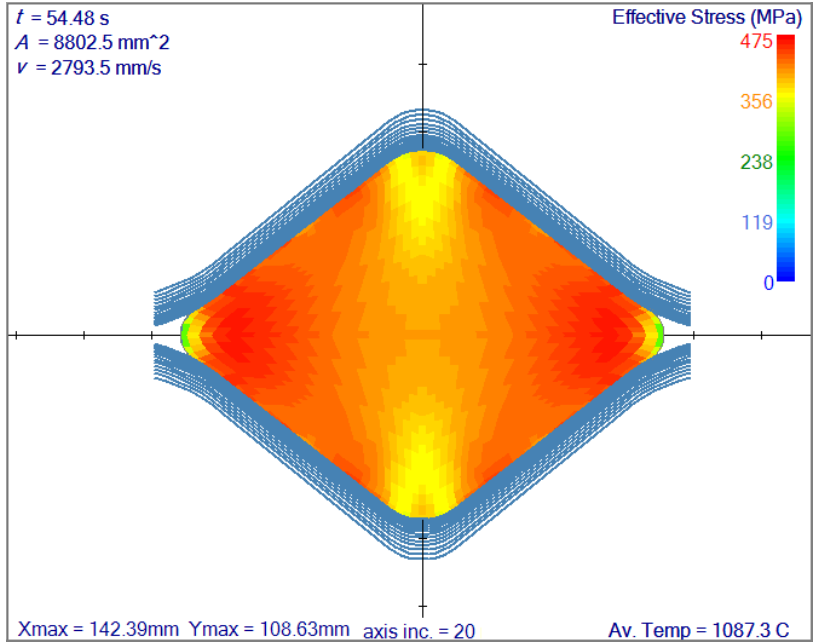

a) 1 st pass

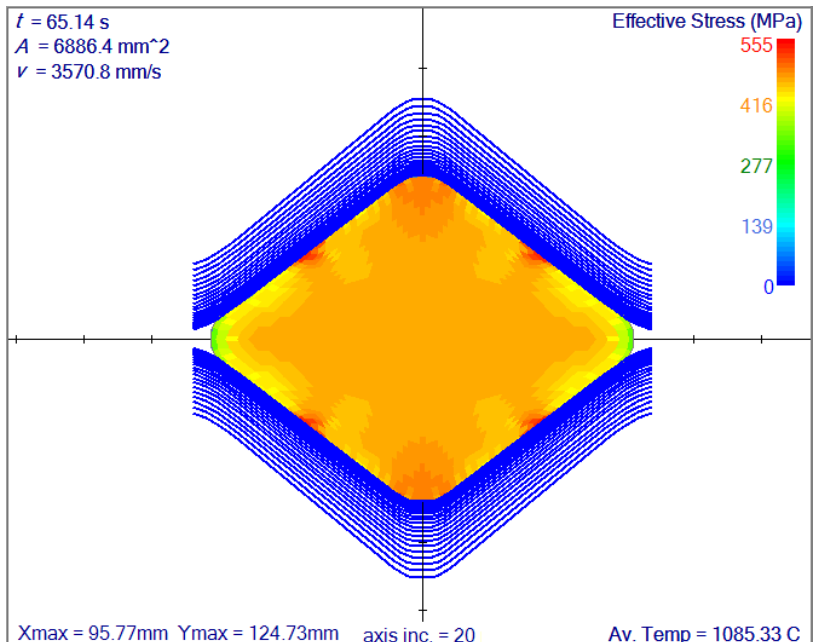

b) 2nd pass

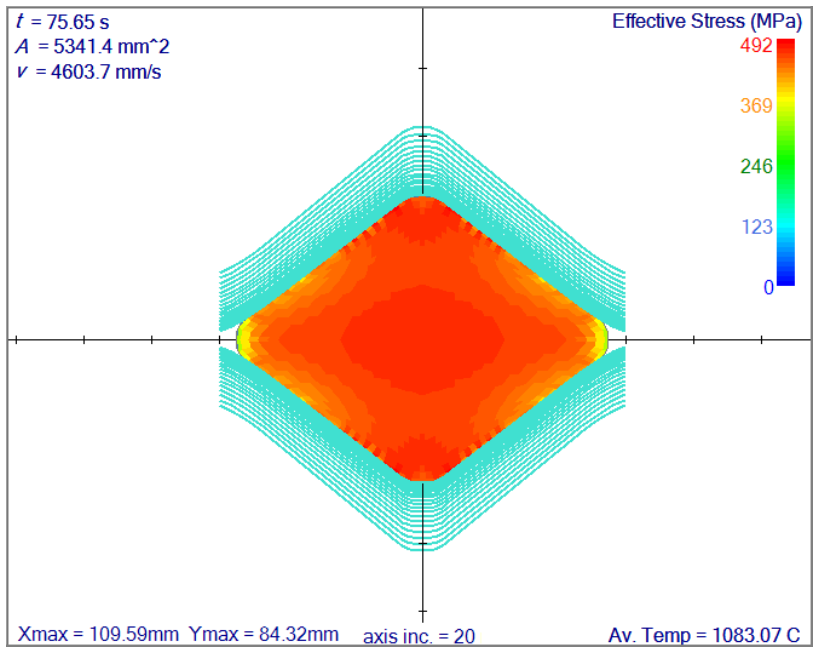

c) 3rd pass

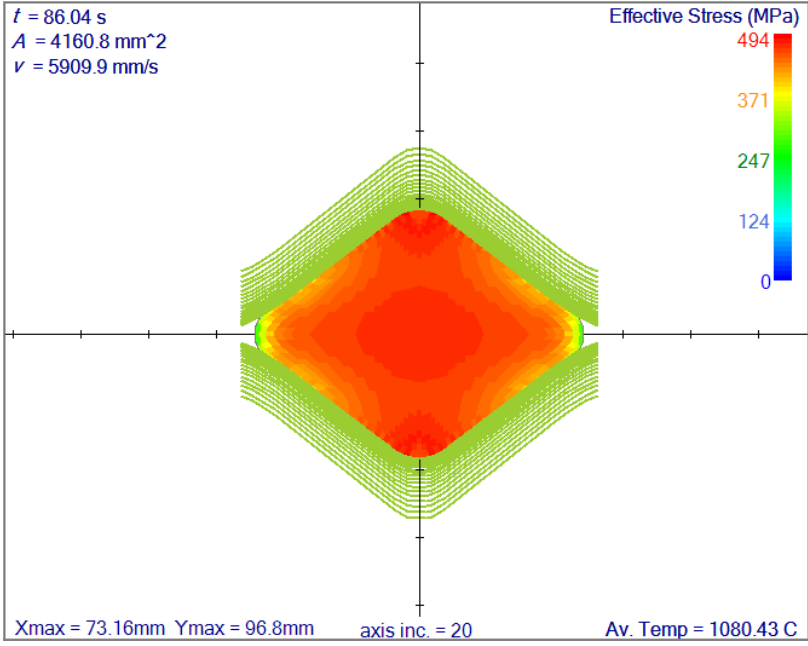

d) 4th pass

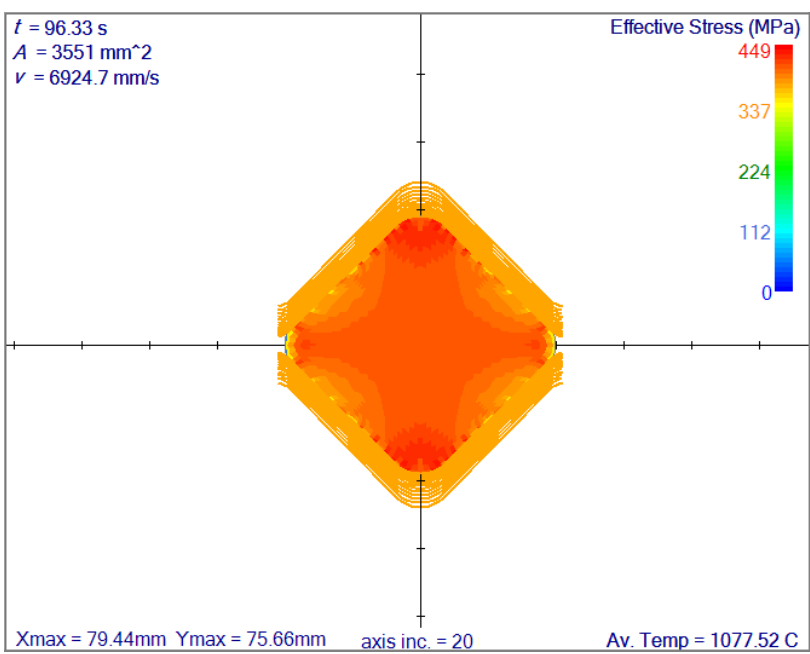

e) 5 th pass

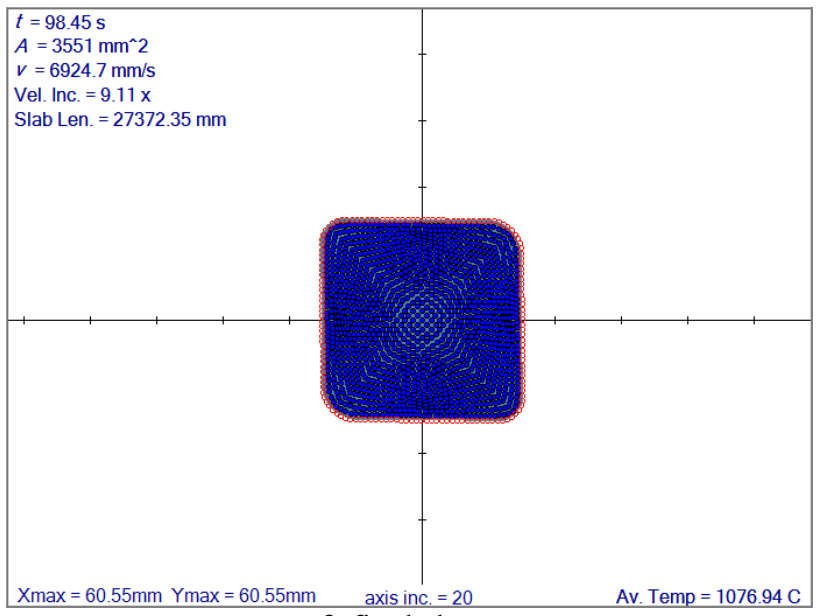

f) final shape

Figure 5. Effective stress field results of the second 5 passes and the final shape. Before the first and after the last pass the billet is rotated 45 degrees and in-between other passes it is rotated 90 degrees.

The simulation results shown in Fig. 4 and Fig. 5 are directly obtained from the user interface of the simulation code after simulation. Results may be seen over one slice at a time, based on its position, until the end of the rolling process. Results in this chapter are shown exactly at 
positions where the slice leaves each rolling stand and all the previously contacting groove lines from the same rolling stand are drawn as well. Except for the first rolling stand, after each pass the slice is rotated and a 6 seconds of rotation time is considered. During the first 5 stands $(800 \mathrm{~mm})$ the billet is rotated 90 degrees, however for the subsequent 5 stands $(650 \mathrm{~mm})$ the rotation is only 45 degrees. In this way, it is possible to consider only the horizontally oriented rolling stands. The increment between the slice positions is $5 \mathrm{~mm}$ when there is a contact. If there is no contact, the incremental distance is increased to $20 \mathrm{~mm}$. Relatively large reduction rates are applied and the maximum effective stress at each pass is calculated to be around $500 \mathrm{MPa}$. At the end of the simulation a $60 \mathrm{~mm}$ square cross section is obtained just like desired. Initially 3 meter long billet at the end becomes 27.372 meters. The slice is also constantly loosing heat either to the air or to the roll during rolling. Therefore, the average temperature is always decreasing.

\section{Conclusions and future work}

In this work a rolling simulation system is explained and applied to reversing rolling mills. A coupled thermomechanical solution procedure is used and the solution is achieved by using a local meshless collocation method with radial basis functions. Simulation results are shown in two sets where in each set billet goes through 5 passes. In between the passes billet is rotated 90 degrees, in between the sets it is rotated 45 degrees. Corresponding grooves are drawn and the results are shown in terms of effective stress.

A macro simulation model is shown here where the shape of the billet may be predicted for each rolling pass. However, this model should also be coupled with the micro scale simulations to get a complete overview of the deformation and material properties of the rolled material.

\section{Acknowledgements}

The authors of this paper are supported by the program group P2-0162, funded by Slovenian Research Agency, and Store Steel (www.store-steel.si) company.

\section{References}

1. J.G. Lenards, Primer on flat rolling (Elsevier, Oxford, 2007)

2. J.G. Lenards, M. Pietrzyk, L. Ceser, Mathematical and physical simulation of the properties of hot rolled products (Elsevier, Oxford, 1999)

3. R. Vertnik, B. Šarler, Comput Math Appl, 51 (2006)

4. R. Vertnik, B. Šarler, Eng Anal Bound Elem, 45 (2014)

5. B. Mavrič, B. Šarler, Inter J Numer Method H, 25 (2015)
6. T. Dobravec, B. Mavrič, B. Šarler, J Comput Phys, 349 (2017)

7. U. Hanoglu, B. Šarler, Comput Struct 194 (2018)

8. S. Kobayashi, S. I. Oh, T. Altan, Metal forming and finite element method (Oxford University Press, New York, 1989).

9. A J. F. Thompson, B. K. Soni, N. P. Weatherill, Handbook of grid generation (CRC, Florida, 1999)

10. Microsoft Corporation, Microsoft Visual Studio Express 2015 for Windows Desktop, version 14.0.23107.0, (2015) 